\title{
Synthesis of sodium silicate-based silica aerogels with graphene oxide by ambient pressure drying
}

\author{
Oznur Kaya Cakmak ${ }^{1}$ [ $\cdot$ Khalil T. Hassan ${ }^{1} \cdot$ Jiabin Wang ${ }^{1} \cdot$ Xiao Han $^{1} \cdot$ Lidija Šiller $^{1}$
}

Accepted: 4 June 2021 / Published online: 12 June 2021

(c) The Author(s) 2021

\begin{abstract}
Here we study how graphene oxide affects silica aerogels and their physical and mechanical properties by examining volume shrinkage, pore volume, surface area and compressive strength of these composite aerogels. Composite aerogels were made through adding different amount of graphene oxide (GO) to sodium silicate precursor by using ambient pressure drying method. Additionally, the chemical composition of the composite aerogels was determined using X-ray diffraction (XRD) and Fourier transform infrared (FTIR) spectroscopy. A rougher structure was observed when the GO loading increased and the characteristic peak of GO in XRD disappeared due to the random distribution of GO within the silica matrix. FTIR spectrum of composite aerogels shows that the relative intensity of silanol groups on the silica matrix have downward tendency with the addition of GO. The specific surface area had maxima with the addition of $0.01 \mathrm{wt} \% \mathrm{GO}$ surface area to $578 \mathrm{~m}^{2} / \mathrm{g}$. The mechanical strength of aerogels was increased, with the loading of GO from $0.0 \mathrm{wt} \%$ to $0.2 \mathrm{wt} \%$, and the compressive modulus increased from $0.02 \mathrm{MPa}$ to $0.22 \mathrm{MPa}$.
\end{abstract}

Keywords Composite aerogels $\cdot \mathrm{GO} \cdot$ Mechanical properties $\cdot$ Physical properties $\cdot$ Waterglass

\section{Introduction}

Silica aerogel, first prepared by Kistler in 1931, is one of the most popular aerogels [1]. Aerogels are porous (up to 99.8\%) nano-structured materials with remarkable properties such as high surface area (up to $1200 \mathrm{~m}^{2} / \mathrm{g}$ ) [2-4], extremely low thermal conductivity (down to $0.01 \mathrm{Wm}^{-1} \mathrm{~K}^{-1}$ ), low sound speed (down to $100 \mathrm{~m} / \mathrm{s}$ ), low refractive index (1.05) and dielectric constant (1.0-2.0) [4-6]. Due to these features, silica aerogels are attractive materials to use in applications such as sensor materials, capacitors, catalysts, thermal insulation, and cosmic dust collection [6]. However, the high production costs and poor mechanical properties are still restricting their full usage [6]. To reduce the production cost of aerogels, the sodium silicate is considered as an inexpensive precursor to make aerogels. Recently, Zhao et al. have reported aerogel powders synthesized from the least expensive precursor, sodium silicate without ion-exchange [7]. The most frequently used method for drying process is

Oznur Kaya Cakmak

O.Kaya2@ncl.ac.uk

1 School of Engineering, Newcastle University, NE1 7RU Newcastle upon Tyne, UK supercritical point drying because of the low surface tension of supercritical fluids, but it involves an expensive equipment [7] that can be dangerous and time consuming [8, 9]. Another method is freeze drying [4], but it is hard to scale up because of the energy intensive requirements [10], and the method needs vacuum for solvent sublimation and low temperatures [11]. Ambient pressure drying (APD) is an alternative way to decrease the cost and energy of production of aerogels [12]. It depends on replacing the original solvent used for gel formation with organic low-surface tension solvents, such as hexane, heptane, octane, etc. [13]. In most APD methods, a surface modification is used to provide a further reduction of capillarity. After surface modification, large quantities of organic solvents are required to remove remnant solvents in the gel [14]. As a result, less energy is consumed; however, it is still very time consuming and costly due to the lengthy solvent exchange process. Recently, Xiao et al. [15] have developed a new approach for APD method. Instead of surface modification and solvent exchange, they treat the wet gel with a combination of sodium bicarbonate solution and TMCS, and carbon dioxide is generated inside the wet silica gel without surface modification. The formation of a bubble of carbon dioxide gas in a pore of the gel opposes capillary pressure [15]. 
To improve the mechanical properties of silica aerogels, there are two methods widely used. One of these is addition of polymers to make the highly cross-linked threedimensional nano-network while the second is incorporation of fibres or nano dopants inside the silica aerogel matrix [16-21].

Graphene is one of the best nanofillers to enhance the comprehensive strength performance of host materials [22]. However, the low dispersion of graphene does not enable it to be dissoluble without the extreme conditions of sonication. Restacking and aggregating of the graphene affect the properties of the final product. Therefore, GO is a much better option because it has oxygen containing groups on the surface [23], which make them a good candidate for use in the above-mentioned applications through chemical functionalization. Nevertheless, in the literature there is still little information about the influence of GO on the silica matrix. The influence of GO on the cellulose aerogel was studied, and the compression strength and Young's modulus of the cellulose aerogels were significantly improved [24]. Guo at al. [25] prepared resorcinol-formaldehyde (RF) aerogels with GO. According to their results, GO improved mechanical properties, and the volume shrinkage of composites was hindered substantially [25]. It has been reported that graphene oxide (GO)/silica composite aerogels, which were fabricated by a sol-gel method using tetraethyl orthosilicate (TEOS), displayed higher mechanical properties compared to pure silica aerogel [26]. Zheng et al. [27] produced graphene oxide (GO)/silica-based composite aerogels via a sol-gel reaction of organosilane precursors in the presence of GOs with silylation treatment. GO treated as cross-linking points to greatly improve the mechanical properties and the thermal stabilities of the network of the composite aerogels. These GO/silica-based composite aerogels can be promising for applications under some harsh and extreme conditions [27]. Lei et al. [28] added GO into silica ( $\mathrm{Si}$ ) aerogels to prepare $\mathrm{GO} / \mathrm{Si}$ composite aerogels, and the compressive modulus was enhanced from $0.238 \mathrm{MPa}$ to $0.394 \mathrm{MPa}$. Furthermore, the composite aerogels showed some toughness improvement compared to the brittleness of pure aerogel. These GO/Si composite aerogels exhibited large surface areas and low thermal conductivity, which are potential for insulation applications. However, Lei et al. [28] used tetraethoxysilane (TEOS) as a silica precursor and the gels were dried by utilising supercritical drying technique, which is expensive. Choi et al. [29] reported in their paper that graphene oxide/sodium silicate $\left(\mathrm{G}-\mathrm{O} / \mathrm{Na}_{2} \mathrm{SiO}_{3}\right)$ nanocomposite which has been produced at room temperature by using $\mathrm{HCl}$ to adjust the $\mathrm{pH}$ level of sols and prepared continuous thin films on silicon wafer. Although the main processing used in their experiments is the same as the one used in this study, drying process is different because they did not use sodium bicarbonate solution as the drying solvent. Instead, all samples were dried at room temperature for $24 \mathrm{~h}$. Sodium ions were not washed out (like in our work, see below) and it was suggested to be a main reason for electrical conductivity of the continuous films. Additionally, there was no study regarding the mechanical properties of $\mathrm{G}-\mathrm{O} / \mathrm{Na}_{2} \mathrm{SiO}_{3}$ nanocomposites. Besides all of these, GO has been applied to many fields as an excellent nano-filler [30]. Zhang et al. [31] developed graphene oxide/silica hybrid aerogel by using the sol-gel method. The hybrid aerogel was produced by adding GO into silica gel display high surface areas and low thermal conductivity [31]. Wang et al. [32] developed GO-ordered mesoporous silica materials with 2D mesoporous structure and large surface area. The as-formed GO/silica aerogel exhibited remarkable efficiency in absorbing toxic heavy metals from wastewater even at low concentrations [32].

In this work, we demonstrated the production of graphene oxide reinforced silica aerogels by using sodium silicate as a precursor and APD method with the sodium bicarbonate solution as the drying solvent (which was suggested previously as a viable alternative [15]). The main purpose is to investigate $\mathrm{GO}$ embedded in the silica matrix to identify the potential of carbon-based materials when used as a nanofiller for silica aerogels. The influences of GO on the mechanical and physical properties of silica aerogel composites were analysed by investigating compressive strength, volume shrinkage, pore volume, chemical composition and surface area of the composite aerogels.

\section{Experimental}

Natural graphite flakes ( $99.8 \%$ purity) and sulphuric acid (98\%) were purchased from VWR. Phosphoric acid (85\%), dihydrogen dioxide $(35 \%)$, potassium permanganate $(99 \%)$, chlorotrimethylsilane ( $\geq 99 \%$ ), hydrochloric acid (37\%), and sodium silicate solution were purchased from Sigma. All reagents were used without further purification. Deionised (DI) water (18 $\mathrm{M} \Omega / \mathrm{cm}$ resistivity) from Nanopore purification water system was used in all experiments. GO was generated with oxidants and mixed acids using modified Hummers method [33] by the oxidation and exfoliation of graphite. Firstly, phosphoric acid $(85 \%, 10 \mathrm{ml})$ was added to sulphuric acid $(98 \%, 50 \mathrm{ml})$ at room temperature by using magnetic stirrer. Then graphite $(1.5 \mathrm{~g})$ and potassium permanganate $(4.08 \mathrm{~g})$ were added to the solution. After three days, dihydrogen dioxide was put in the solution drop by drop carefully to cease reaction and the solution's colour turned into bright yellow. The precipitates of this solution were put in the membrane and washed with DI water (the water in beaker with membrane was exchanged regularly each day over a period of $\sim 2$ weeks) to reach the $\mathrm{pH} 7$. Lastly, precipitates were dried at $70^{\circ} \mathrm{C}$ and then dispersed in DI water to generate graphene oxide [34]. The silicon 
source, which was $6 \mathrm{ml}$ sodium silicate as purchased without any further purification and $30 \mathrm{ml}$ distilled water were mixed to make the silica precursor solution. The molar ratio of the sodium silicate and distilled water was 1:5. GO solutions were prepared by mixing them with ultrasonic probe for $15 \mathrm{~min}$. Graphene oxide weights $22 \mathrm{mg}(0.05 \mathrm{wt} \%), 44 \mathrm{mg}$ $(0.1 \mathrm{wt} \%)$, and $89 \mathrm{mg}(0.2 \mathrm{wt} \%)$ in silica aerogel composites.

\subsection{Synthesis of $\mathrm{SiO}_{2}$ and $\mathrm{Si}-\mathrm{GO}$ wet gels}

Sol gel technique was applied to make silica gels due to hydrolysis of sodium silicate in distilled water. $\mathrm{HCl}(5 \mathrm{ml})$ as a catalyst was added to silicate solution to turn sol into gel within $8 \mathrm{~min}$. First day, the gel was washed with water and then sodium bicarbonate solution was used in a solvent exchange. After using TMCS for carbon dioxide generation, the gel was washed by distilled water again to remove $\mathrm{NaCl}$, which came from the reaction between TMCS and $\mathrm{NaHCO}_{3}$. The last day, water was replaced with ethanol. Finally, the gel was dried on the heater at $70{ }^{\circ} \mathrm{C}$ for $24 \mathrm{~h}$. The prepared pure monolithic silica aerogel was then ready for characterization.

Figure 1 shows the schematic diagram of the preparation of Si-GO composite gels. Firstly, two beakers were prepared. One of them included GO solution $(12 \mathrm{ml}$ distilled water $+\mathrm{GO}$ ), the other one had $6 \mathrm{ml}$ water glass as a silica precursor and $18 \mathrm{ml}$ distilled water. First beaker was added into the second beaker and resulting $6 \mathrm{ml}$ water glass, 30 $\mathrm{ml}$ water and GO $(0.05 \mathrm{wt} \%, 0.1 \mathrm{wt} \%, 0.2 \mathrm{wt} \%)$ stirred in sonication bath for $30 \mathrm{~min}$. Then the solution was left for one day to form sol. Next day, the addition of HCI turned the sol into gel and the remaining processes to produce final
$\mathrm{Si} / \mathrm{GO}$ composite aerogels were the same as the production of a monolithic pure silica aerogel.

\subsection{Characterization}

X-ray diffraction (XRD) was carried out using PanalyticalX'pert Pro diffractometer with an X'Celerator area detector, and $\mathrm{Cu} \mathrm{K} \alpha \mathrm{X}$-rays were used to investigate the crystalline and amorphous morphology. Scanning electron microscopy (SEM) measurements were performed on a Philips XL30 ESEM FEG environmental scanning electron microscope. Coulter SA 3100 Surface Area and Pore Size Analyzer was used to determine the nitrogen adsorption isotherms. The specific surface area of the aerogels was determined by Brunauer-Emmet Teller (BET) method. Uniaxial compression tests were carried out with ASTM D695 test method.

\section{Results and discussion}

In this study, the recently developed APD mechanism using sodium bicarbonate solution to decrease capillary pressure has been examined [15]. The wet gel was exposed to a combination of sodium bicarbonate solution and TMCS that generates carbon dioxide within the bulk of silica and silica composite gels without surface modification. The carbon dioxide that is trapped in the wet gel opposes the capillary pressure, the pore shrinkage and collapse during the drying step is prevented due to this decrease of capillary pressure. The effect of GO on the structural properties of composite aerogels is studied by adding a different amount of GO. The sol-gel method was
Fig. 1 Schematic diagram of Si-GO composite aerogels

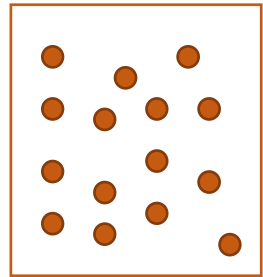

Water glass $+\mathrm{GO}+\mathrm{DI}$ water

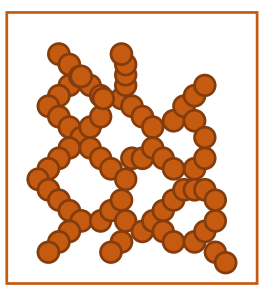

Dried at $70{ }^{\circ} \mathrm{C}$
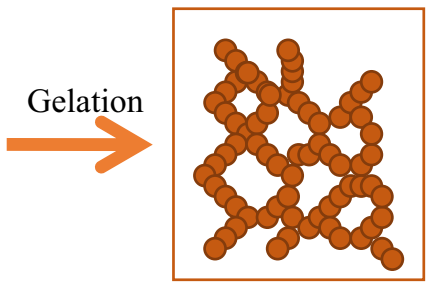

Si-GO wet gel

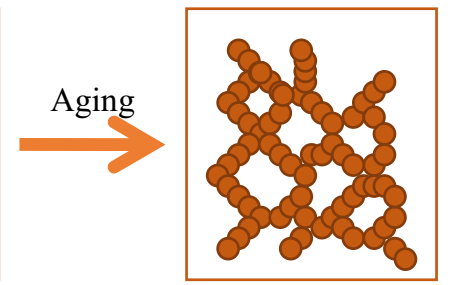

Aged by DI water
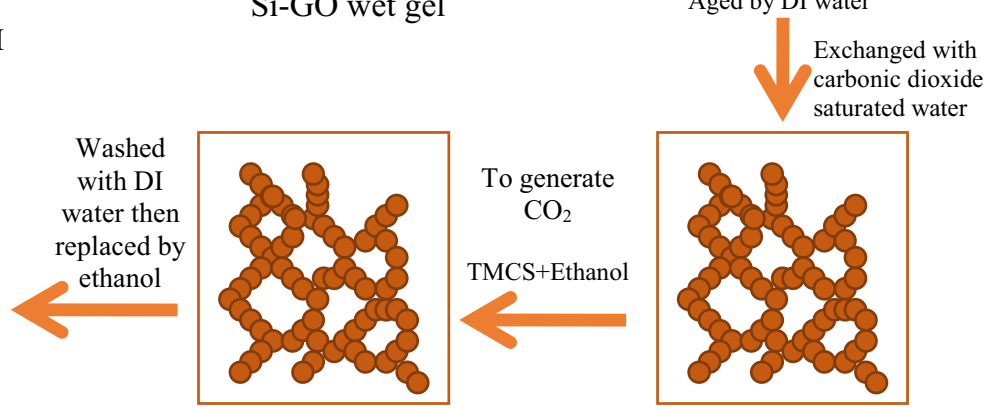
water 
used to incorporate graphene oxide solution at the stage of gel formation to ensure homogeneous dispersion of graphene oxide solution within the silica network by ultrasonic agitation. Silica aerogel composite which includes the highest value of GO $(0.2 \mathrm{wt} \%)$ required $30 \mathrm{~min}$ to form hydrogel. The composite aerogel with the lowest GO wt $\%$ content $(0.05)$ and the monolithic silica aerogel are gelated in $7 \mathrm{~min}$. The delayed gelation time with the higher content of GO in gel could be probably because of the saturated adsorption of GO sheets in the silica matrix; therefore, the hydrolysis and condensation reactions between siloxane chains were inhibited and gelation time retarded. The other possible reason could be that GO reacts with hydrolysed silica particles.

The XRD data for pure GO, and silica aerogels and composites are shown in Fig. $2 \mathrm{a}$ and b, respectively. A sharp diffraction peak at $2 \theta=11.3^{\circ}$ (see Fig. 2a) is the characteristic peak of the pristine GO $[35,36]$. At the same time, the characteristic peak has disappeared, see Fig. 2b, and an amorphous diffraction peak at $2 \theta=22^{\circ}$ is observed which is known the characteristic peak of silica aerogel. The characteristic peak of pure GO is missing in composites because of the random distribution of GO within the silica matrix [37].

The surface morphology obtained by SEM of the pristine GO and the composite aerogels is shown in Fig. 3. The pristine GO are large and smooth nanosheets with some wrinkles. Many oxygenated functional groups such as hydroxyl groups and carboxyl groups on the surface of the GO [38] could be advantageous for dispersion in the silica aerogel matrix and interaction with the silica matrix. The presence of GO nanosheets does not hinder the integrity of composite aerogels. The prepared composite aerogels have a rough structure and become coarser as the GO loading increases (see Fig. 3).
The FTIR spectrum of (a) GO and (b) Si/GO composite aerogels are shown in Fig. 4. The FTIR spectrum of GO showed the characteristic peaks of approximately 3400 and $1740 \mathrm{~cm}^{-1}$ corresponding to $\mathrm{O}-\mathrm{H}$ and $\mathrm{C}=\mathrm{O}$ stretching frequencies of the $-\mathrm{COOH}$ group, respectively [38]. Oxygen containing functional groups which are in the graphene oxide nanosheets do not appear when the GO nanosheets are added into the silica matrix. This could be because of the chemical interaction between the silica particles and GO surfaces. In the FTIR spectrum of pure silica aerogels, the peak centred at $1087 \mathrm{~cm}^{-1}, 807 \mathrm{~cm}^{-1}$, and $473 \mathrm{~cm}^{-1}$ are the main characteristic peaks which are $\mathrm{Si}-\mathrm{O}-\mathrm{Si}$ bands of silica aerogels [39]. Silanol groups are seen at $950 \mathrm{~cm}^{-1}$ [39]. The peak around $3000 \mathrm{~cm}^{-1}$ is assigned to alkyl groups due to the remaining non-hydrolysed ethoxy silane groups or residual slight solvent $[40,41]$. With the addition of GO nanosheets, the relative intensity of silanol groups at $1083 \mathrm{~cm}^{-1}$ on the silica matrix decreases. Another possibility is that because the oxygen-containing groups due to moisture, for instance $\mathrm{O}-\mathrm{H}$ and $\mathrm{C}-\mathrm{OH}$ on the graphene oxide sheets can also consume the silanol groups of silica nanoparticles in the sol-gel process, the relative intensity of $\mathrm{Si}-\mathrm{O}-\mathrm{Si}$ groups in composite aerogels become weaker [28]. However, the relative intensity of silanol groups for $\mathrm{Si} / \mathrm{GO}$ composite aerogel with $0.2 \mathrm{wt} \%$ GO shows slight increase.

Table 1 shows the pore properties of aerogels. The specific surface area of pure silica aerogel $\left(550 \mathrm{~m}^{2} / \mathrm{g}\right)$ is higher than the $\mathrm{Si} / \mathrm{GO}$ composite aerogels with $0.2 \mathrm{wt} \% \mathrm{GO}$ due to the slight increase of total pore volume and small size of pores. The specific surface area of $\mathrm{Si} / \mathrm{GO}$ composite aerogel with $0.1 \mathrm{wt} \%$ GO has the highest specific surface area, the reason for which can be relatively small-sized pores and relatively high total pore volume. The total pore volume of silica composite aerogels slightly increases, which can be

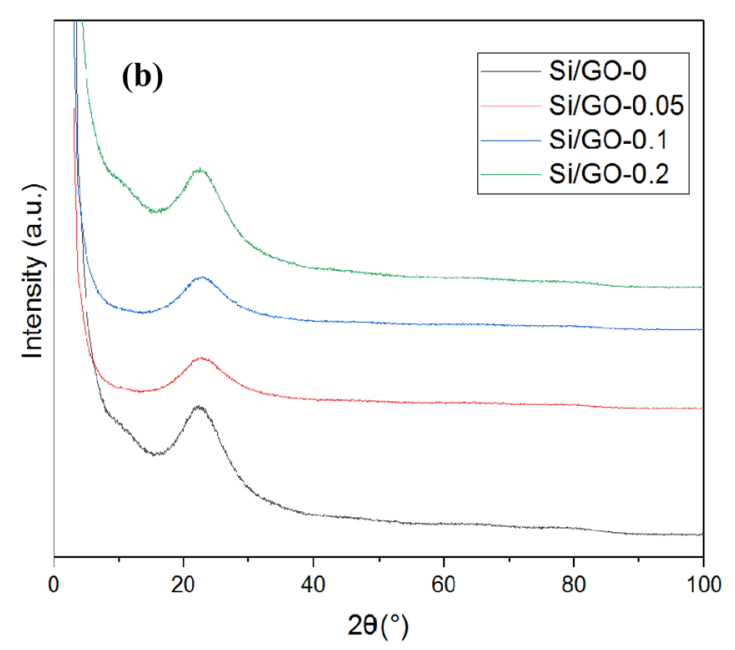

Fig. 2 XRD patterns of $\mathbf{a}$ the pristine GO and $\mathbf{b}$ Si-GO composite aerogels 

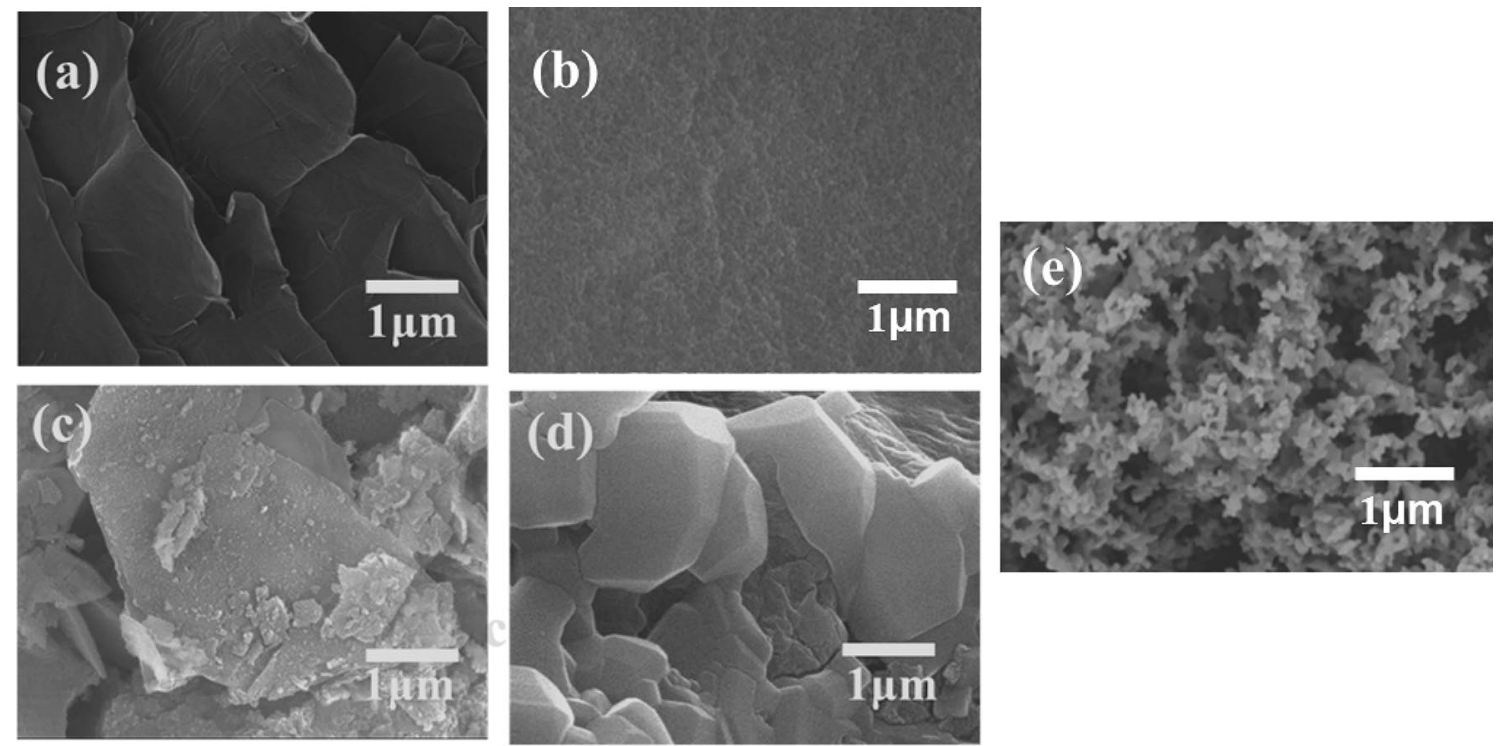

Fig. 3 SEM micrographs of a GO, b pure silica aerogel and Si-GO composite aerogels with different loading of GO c 0.5 wt $\%$, d 0.1 wt $\%$, e 0.2 wt $\%$

(a)

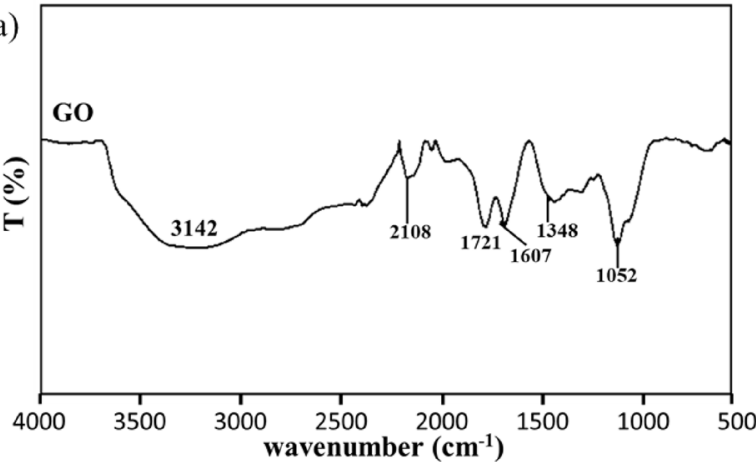

(b)

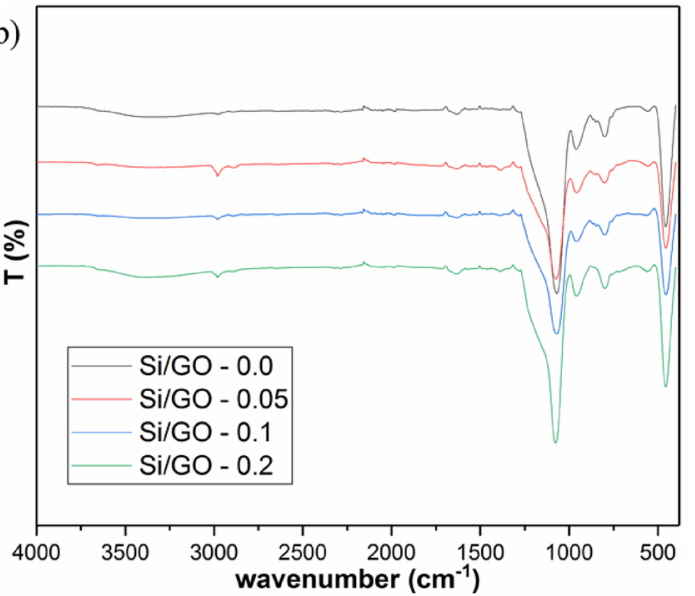

Fig. 4 FTIR spectrum of $\mathbf{a}$ GO and $\mathbf{b}$ pure silica aerogels and $\mathrm{Si} / \mathrm{GO}$ composite aerogels

Table 1 The pore properties of monolithic and composite Si/GO aerogels

\begin{tabular}{lllll}
\hline Samples & $\begin{array}{l}\mathrm{SiO}_{2}-\mathrm{GO} \\
(0.0)\end{array}$ & $\begin{array}{l}\mathrm{SiO}_{2}-\mathrm{GO} \\
(0.05)\end{array}$ & $\begin{array}{l}\mathrm{SiO}_{2}-\mathrm{GO} \\
(0.1)\end{array}$ & $\begin{array}{l}\mathrm{SiO}_{2}-\mathrm{GO} \\
(0.2)\end{array}$ \\
\hline $\mathrm{S}_{\text {BET }}\left(\mathrm{m}^{2} / \mathrm{g}\right)$ & 550 & 563 & 578 & 541 \\
$\mathrm{~V}_{\text {total }}\left(\mathrm{cm}^{3} / \mathrm{g}\right)$ & 1.08 & 0.94 & 0.98 & 1.05 \\
$\mathrm{R}_{\text {average }}(\mathrm{nm})$ & 8.33 & 6.81 & 6.45 & 11.32 \\
\hline
\end{tabular}

because of the increment of mesopore volume. However, $\mathrm{Si} / \mathrm{GO}$ composite aerogel with $0.2 \mathrm{wt} \%$ GO has the lowest specific surface area, due to the increase in the amount of graphene oxide, it may not be dispersed well in the silica matrix as a result it may GO could restack or agglomerate and therefore lead to decrease of the specific surface area

The nitrogen adsorption - desorption isotherms for monolithic and composite aerogels are shown in Fig. 5. The isotherms of $\mathrm{Si} / \mathrm{GO}$ composite aerogels are typical IUPAC type IV with hysteresis loops of type $\mathrm{H} 2$ at the high relative pressure range because of the capillary condensation in mesopores [42].

The mechanical strength of aerogels with GO addition is enhanced compared to that of the monolithic silica aerogel. As shown in Fig. 6, the pure silica aerogel showed compressive strength of $0.02 \mathrm{MPa}$. On the contrary, the 

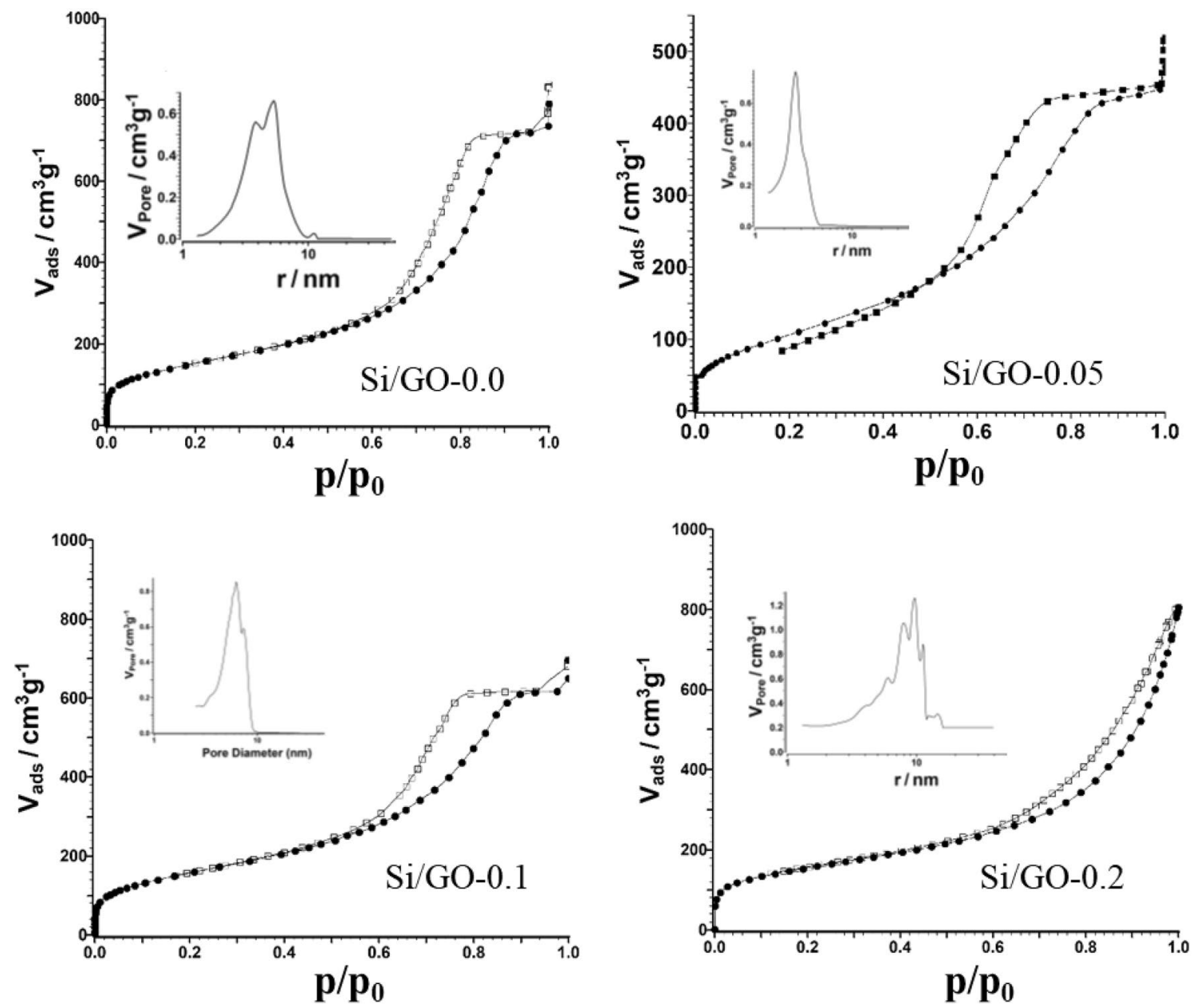

Fig. 5 Nitrogen adsorption-desorption isotherms and pore-size distributions of the monolithic and composite Si/GO aerogels

Fig. 6 The compressive strength of the monolithic and composite $\mathrm{Si} / \mathrm{GO}$ aerogels

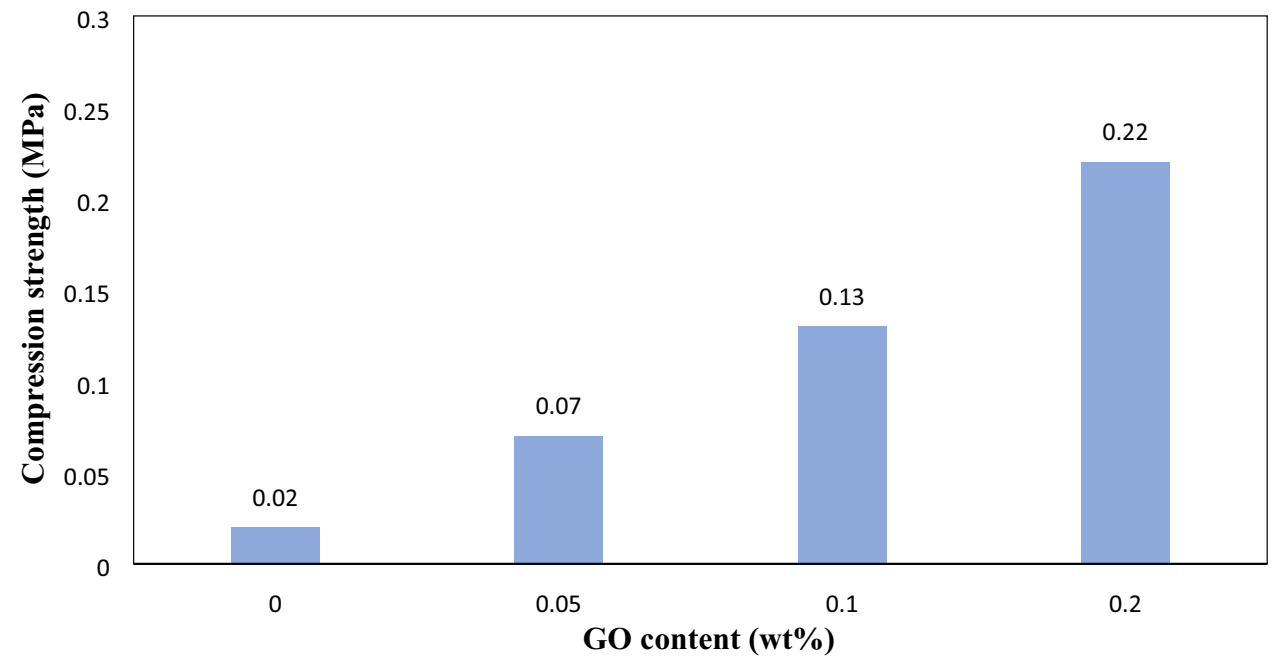

$\mathrm{GO} /$ silica composite aerogels can resist higher stress. The compressive strength of $\mathrm{GO} /$ silica composite aerogels with $0.05 \mathrm{wt} \%, 0.1 \mathrm{wt} \%, 0.2 \mathrm{wt} \%$ is $0.07 \mathrm{MPa}, 0.13 \mathrm{MPa}$,
$0.22 \mathrm{MPa}$, respectively. That the addition of GO can improve mechanical strength of the composite aerogels is based on two aspects. Firstly, the existence of GO can avoid further 
expansion of small cracks in aerogels because of its high mechanical strength. Secondly, the GO nanosheets can prevent the crack to further propagate, which can deplete the parts of fracture energy. This is convenient for the enhancement of mechanical strength. Lei et al. [28] reported that the silica aerogel monoliths mixed with GO were fabricated by using supercritical drying method. The compressive strength of composite aerogels is higher than that of aerogels in our study. One of the reasons of the higher compressive strength can be the usage of TEOS as a silica precursor because TEOS provides the control of porosity. Because of that, a stiffer structure can be obtained [43]. The other reason can be the supercritical drying method, which facilitates a much more uniform structure and produced aerogel, exhibits better mechanical properties [44]. Choi et al. [29] reported in their paper that graphene oxide/sodium silicate $\left(\mathrm{G}-\mathrm{O} / \mathrm{Na}_{2} \mathrm{SiO}_{3}\right)$ nanocomposite prepared as in this work but without washing step of sodium and with different drying step, are excellent method for thin films preparation at ambient conditions when good electrical conductivity (due to sodium ions) is required.

However, novel APD method [15] applied in this work with water glass as a silica precursor are much cheaper than supercritical drying method and TEOS as a silica precursor. In addition, novel APD method and water glass as a silica precursor can be produced at large scale without presence of sodium ions.

\section{Conclusions}

In this work, the low-cost bicarbonate APD drying process recently published [14] has been applied in preparation of uniformly dispersed GO/Si composite aerogels. The prepared aerogels have a rough structure which becomes coarser as the GO loading is increased. XRD results showed that the characteristic peaks of GO were covered, and only silicon aerogel amorphous structure occurred. According to BET results, the specific surface area of Si/GO composite aerogel with $0.1 \mathrm{wt} \% \mathrm{GO}$ has the highest surface area of $578 \mathrm{~m}^{2} / \mathrm{g}$. FTIR results demonstrated that with the addition of GO nanosheets, the relative intensity of silanol groups on the silica matrix decreases. The mechanical strength of aerogels has increased with the addition of GO. This study shows a right step towards a cost effectiveness for a largescale production of these composites.

Acknowledgements O.C. is thankful to Turkish government for the scholarship.

\section{Declarations}

Conflict of interest The authors declare no conflict of interest.
Open Access This article is licensed under a Creative Commons Attribution 4.0 International License, which permits use, sharing, adaptation, distribution and reproduction in any medium or format, as long as you give appropriate credit to the original author(s) and the source, provide a link to the Creative Commons licence, and indicate if changes were made. The images or other third party material in this article are included in the article's Creative Commons licence, unless indicated otherwise in a credit line to the material. If material is not included in the article's Creative Commons licence and your intended use is not permitted by statutory regulation or exceeds the permitted use, you will need to obtain permission directly from the copyright holder. To view a copy of this licence, visit http://creativecommons.org/licenses/by/4.0/.

\section{References}

1. S. Kistler, Coherent expanded aerogels. Rub. Chem. Technol. 5(4), 600-603 (1932). https://doi.org/10.5254/1.3539386

2. J. Fricke, Aerogels - highly tenuous solids with fascinating properties. J. Non-Crystall. Sol. 100(1-3), 169-173 (1988). https://doi.org/10.1016/0022-3093(88)90014-2

3. H. Maleki, Recent advances in aerogels for environmental remediation applications: A review. Chem. Eng. J. 300, 98-118 (2016)

4. A. Soleimani Dorcheh, M. Abbasi, Silica aerogel; synthesis, properties, and characterization. J. Mater. Proc. Technol. 199(13), 10-26 (2008). https://doi.org/10.1016/j.jmatprotec.2007.10. 060

5. G. Kim, S. Hyun, H. Park, Synthesis of Low-Dielectric Silica Aerogel Films by Ambient Drying. J. Am. Ceramic Soc. 84(2), 453-455 (2004). https://doi.org/10.1111/j.1151-2916.2001. tb00677.x

6. J. Gurav, I. Jung, H. Park, E. Kang, D. Nadargi (2010) Silica Aerogel: Synthesis and Applications. Journal Of Nanomaterials. https://doi.org/10.1155/2010/409310

7. S. Zhao, A. Stojanovic, E. Angelica, O. Emery, D. Rentsch, R. Pauer, M. Koebel, W. Malfait (2020). Phase transfer agents facilitate the production of superinsulating silica aerogel powders by simultaneous hydrophobization and solvent- and ionexchange. Chemical Engineering Journal, 381, 122421

8. T. Zhou, X. Cheng, Y. Pan, C. Li, L. Gong, H. Zhang, Mechanical performance and thermal stability of glass fiber reinforced silica aerogel composites based on co-precursor method by freeze drying. Appl. Surf. Sci. 437, 321-328 (2018)

9. A. Anderson, M. Carroll, E. Green, J. Melville, M. Bono, Hydrophobic silica aerogels prepared via rapid supercritical extraction. J. Sol-Gel Sci. Technol. 53(2), 199-207 (2009). https://doi.org/10.1007/s10971-009-2078-z

10. L. Ren, S. Cui, F. Cao, Q. Guo, An Easy Way To Prepare Monolithic Inorganic Oxide Aerogels. Angewandte Chemie 126(38), 10311-10313 (2014). https://doi.org/10.1002/ange.201406387

11. C. Siligardi, P. Miselli, E. Francia, M. Lassinantti Gualtieri, Temperature-induced microstructural changes of fiber-reinforced silica aerogel (FRAB) and rock wool thermal insulation materials: A comparative study. Energy And Buildings 138, 80-87 (2017). https://doi.org/10.1016/j.enbuild.2016.12.022

12. S. Prakash, C. Brinker, A. Hurd, S. Rao, Silica aerogel films prepared at ambient pressure by using surface derivatization to induce reversible drying shrinkage. Nature 374(6521), 439-443 (1995). https://doi.org/10.1038/374439a0

13. A. Parvathy Rao, A. Venkateswara Rao, G. Pajonk, P. Shewale, Effect of solvent exchanging process on the preparation of the hydrophobic silica aerogels by ambient pressure drying method using sodium silicate precursor. J. Mater. Sci. 42(20), 84188425 (2007). https://doi.org/10.1007/s10853-007-1788-2 
14. T. Wei, T. Chang, S. Lu, Y. Chang, Preparation of monolithic silica aerogel of low thermal conductivity by ambient pressure drying. J. Am. Ceramic Soc. 90(7), 2003-2007 (2007).https:// doi.org/10.1111/j.1551-2916.2007.01671.x

15. X. Han, K. Hassan, A. Harvey, D. Kulijer, A. Oila, M. Hunt, L. Šiller, Bioinspired synthesis of monolithic and layered aerogels. Adv. Mater. 30(23), 1706294 (2018). https://doi.org/10.1002/ adma. 201706294

16. L. Capadona, M. Meador, A. Alunni, E. Fabrizio, P. Vassilaras, N. Leventis, Flexible, low-density polymer crosslinked silica aerogels. Polymer 47(16), 5754-5761 (2006). https://doi.org/ 10.1016/j.polymer.2006.05.073

17. S. Mulik, C. Sotiriou-Leventis, G. Churu, H. Lu, N. Leventis, Cross-Linking 3D Assemblies of Nanoparticles into Mechanically Strong Aerogels by Surface-Initiated Free-Radical Polymerization. Chem. Mater. 20(15), 5035-5046 (2008). https://doi. org $/ 10.1021 / \mathrm{cm} 800963 \mathrm{~h}$

18. M. Meador, S. Vivod, L. McCorkle, D. Quade, R. Sullivan, L. Ghosn et al., Reinforcing polymer cross-linked aerogels with carbon nanofibers. J. Mater. Chem. 18(16), 1843 (2008). https://doi. org $/ 10.1039 / \mathrm{b} 800602 \mathrm{~d}$

19. M. Meador, A. Weber, A. Hindi, M. Naumenko, L. McCorkle, D. Quade et al., Structure - Property Relationships in Porous 3D Nanostructures: Epoxy-Cross-Linked Silica Aerogels Produced Using Ethanol as the Solvent. ACS Appl. Mater. Interfaces. 1(4), 894-906 (2009). https://doi.org/10.1021/am900014z

20. H. Maleki, L. Durães, A. Portugal, An overview on silica aerogels synthesis and different mechanical reinforcing strategies. Journal Of Non-Crystalline Solids 385, 55-74 (2014). https://doi.org/10. 1016/j.jnoncrysol.2013.10.017

21. H. Maleki, L. Durães, B. Costa, R. Santos, A. Portugal, Design of multifunctional magnetic hybrid silica aerogels with improved properties. Microporous And Mesoporous Materials 232, 227-237 (2016). https://doi.org/10.1016/j.micromeso.2016.06.025

22. A. Geim, K. Novoselov, The rise of graphene. Nat. Mater. 6(3), 183-191 (2007). https://doi.org/10.1038/nmat1849

23. W. Gao, L. Alemany, L. Ci, P. Ajayan, New insights into the structure and reduction of graphite oxide. Nat. Chem. 1(5), 403-408 (2009). https://doi.org/10.1038/nchem.281

24. J. Zhang, Y. Cao, J. Feng, P. Wu, Graphene-oxide-sheet-induced gelation of cellulose and promoted mechanical properties of composite aerogels. J. Phys. Chem. 116(14), 8063-8068 (2012). https://doi.org/10.1021/jp2109237

25. K. Guo, H. Song, X. Chen, X. Du, L. Zhong, Graphene oxide as an anti-shrinkage additive for resorcinol-formaldehyde composite aerogels. Phys. Chem. Chem. Phys. 16(23), 11603-11608 (2014). https://doi.org/10.1039/c4cp00592a

26. L. Hong-li, H. Xiang, L. Hong-yan, L. Jing, L. Ya-jing, Novel GO/ silica composite aerogels with enhanced mechanical and thermal insulation properties prepared at ambient pressure. Ferro Electrics 528(1), 15-21 (2018)

27. Z. Zheng, Y. Zhao, J. Hu, H. Wang, Flexible, Strong, Multifunctional Graphene Oxide/Silica-Based Composite Aerogels via a Double-Cross-Linked Network Approach. ACS Appl. Mater. Interfaces. 12(42), 47854-47864 (2020)

28. Y. Lei, Z. Hu, B. Cao, X. Chen, H. Song, Enhancements of thermal insulation and mechanical property of silica aerogel monoliths by mixing graphene oxide. Mater. Chem. Phys. 187, 183-190 (2017)

29. J. Choi, H. Lee, S. An, Synthesis of a graphene oxide/sodium silicate nanocomposite using sodium silicate solution. RSC Advances 5(48), 38742-38747 (2015)

30. Y. Ma, H. Di, Z. Yu, L. Liang, L. Lv, Y. Pan, Y. Zhang, D. Yin, Fabrication of silica-decorated graphene oxide nanohybrids and the properties of composite epoxy coatings research. Appl. Surf. Sci. 360, 936-945 (2016)

31. M. Zhang, Q. Xiao, C. Chen, L. Li, W. Yuan, Developing a heatinsulating composite phase change material with light-to-thermal conversion performance from graphene oxide/silica hybrid aerogel. Applied Thermal Engineering, 174, 115303 (2020)

32. X. Wang, Y. Pei, M. Lu, X. Lu, X. Du, Highly efficient adsorption of heavy metals from wastewaters by graphene oxide-ordered mesoporous silica materials. Journal of Materials Science 50(5), 2113-2121 (2015)

33. W. Hummers, R. Offeman, Preparation of graphitic oxide. Journal Of The American Chemical Society 80(6), 1339-1339 (1958). https://doi.org/10.1021/ja01539a017

34. J. Wang, E. Salihi, L. Šiller, Green reduction of graphene oxide using alanine. Materials Science And Engineering: C 72, 1-6 (2017). https://doi.org/10.1016/j.msec.2016.11.017

35. T. Kuila, S. Bose, P. Khanra, A. Mishra, N. Kim, J. Lee, A green approach for the reduction of graphene oxide by wild carrot root. Carbon 50(3), 914-921 (2012). https://doi.org/10.1016/j.carbon. 2011.09.053

36. K. Guo, Z. Hu, H. Song, X. Du, L. Zhong, X. Chen, Low-density graphene/carbon composite aerogels prepared at ambient pressure with high mechanical strength and low thermal conductivity. RSC Advances 5(7), 5197-5204 (2015). https://doi.org/10.1039/c4ra0 $8800 \mathrm{j}$

37. C. Petit, T. Bandosz, Graphite Oxide/Polyoxometalate Nanocomposites as Adsorbents of Ammonia. The Journal Of Physical Chemistry C 113(9), 3800-3809 (2009). https://doi.org/10.1021/ jp8097044

38. E. Aliyev, V. Filiz, M. Khan, Y. Lee, C. Abetz, V. Abetz (2019). Structural Characterization of Graphene Oxide: Surface Functional Groups and Fractionated Oxidative Debris. Nanomaterials, $9(8)$, p. 1180

39. M. Ocaña, V. Fornés, C. Serna, The variability of the infrared powder spectrum of amorphous $\mathrm{SiO} 2$. Journal Of Non-Crystalline Solids 107(2-3), 187-192 (1989). https://doi.org/10.1016/00223093(89)90461-4

40. L. Zhong, X. Chen, H. Song, K. Guo, Z. Hu, Highly flexible silica aerogels derived from methyltriethoxysilane and polydimethylsiloxane. New Journal Of Chemistry 39(10), 7832-7838 (2015). https://doi.org/10.1039/c5nj01477h

41. B. Orel, R. Ješe, A. Vilčnik, U. Štangar, Hydrolysis and Solvolysis of Methyltriethoxysilane Catalyzed with $\mathrm{HCl}$ or Trifluoroacetic Acid: IR Spectroscopic and Surface Energy Studies. Journal Of Sol-Gel Science And Technology 34(3), 251-265 (2005). https:// doi.org/10.1007/s10971-005-2522-7

42. K. Sing, Reporting physisorption data for gas/solid systems with special reference to the determination of surface area and porosity (Recommendations 1984). Pure And Applied Chemistry 57(4), 603-619 (1985). https://doi.org/10.1351/pac198557040603

43. M. Shahzamani, R. Bagheri, M. Masoomi, Synthesis of silicapolybutadiene hybrid aerogels: The effects of reaction conditions on physical and mechanical properties. J. Non-Cryst. Solids 452, 325-335 (2016)

44. K. Obrey, K. Wilson, D. Loy, Enhancing mechanical properties of silica aerogels. J. Non-Cryst. Solids 357(19-20), 3435-3441 (2011)

Publisher's Note Springer Nature remains neutral with regard to jurisdictional claims in published maps and institutional affiliations. 